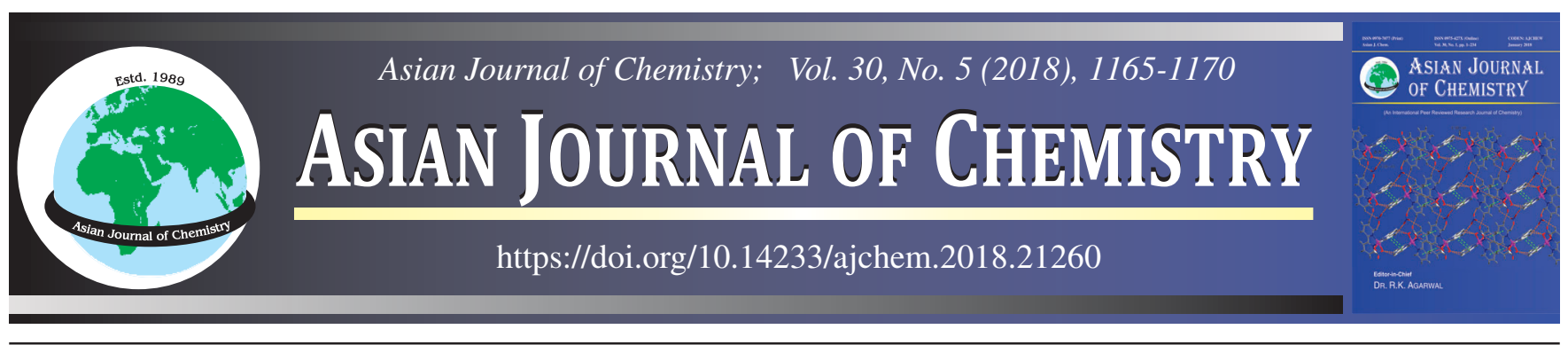

\title{
Highly Effective Opening of Epoxides with Aromatic Amines in Presence of $\boldsymbol{\beta}$-Cyclodextrin in Water
}

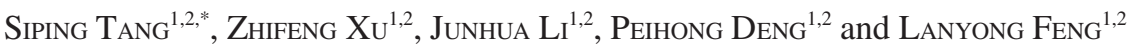

${ }^{1}$ Department of Chemistry and Material Science, Hengyang Normal University, Hengyang 421008, Hunan Province, P.R. China

${ }^{2}$ Key Laboratory of Functional Organometallic Materials of Hunan Province, Hengyang 421008, Hunan Province, P.R. China

*Corresponding author: Fax: +86 734 3456099; Tel: +86 734 8450056; E-mail: tsp00@163.com

Received: 30 January 2018;

Accepted: 9 March 2018;

Published online: 29 March 2018;

AJC-18859

This paper reports a simple, mild, and convenient method to synthesize $\beta$-amino alcohols. These $\beta$-amino alcohols were achieved in excellent yields (80-96\%) by ring-opening reaction of oxiranes with aromatic amines catalyzed by $\beta$-cyclodextrin at $30{ }^{\circ} \mathrm{C}$ in water. $\beta$-cyclodextrin as the catalyst greatly accelerated the reactions and can be recovered and reused.

Keywords: Oxiranes, $\beta$-Cyclodextrin, $\beta$-Amino alcohols, Ring-opening

\section{INTRODUCTION}

$\beta$-Amino alcohols are versatile intermediates in the synthesis of a wide range of biologically active natural and synthetic products such as unnatural amino acids [1,2] and chiral auxiliaries [3]. The classical approach for the preparation of $\beta$-amino alcohols involves the ring opening of epoxides at elevated temperatures in an excess amount of amines [4]. These reactions are accompanied by poor regioselectivity and long reaction time. To avoid these drawbacks, other methods were reported recently, which involved the use of metal amides [5-7], metal triflates [8-10], metal halides [11-14], organobismuth triflate complex [15], copper(II) tetrafluoroborate [16], ionic liquid [17], phosphomolybdic acid- $\mathrm{Al}_{2} \mathrm{O}_{3}[18]$, thiourea [19] and $\mathrm{H} \beta$ zeolite [20]. However, most of these methods involve the use of expensive reagents, air- and/or moisture-sensitive catalysts, hazardous organic solvents and suffering from poor regioselectivity. In view of these limitations, inexpensive, environmentally benign and highly efficient catalysts are expected to be developed for the ring-opening of epoxides directly with anilines.

Having hydrophobic cavities, cyclodextrins form hostguest complexes with substrates via non-covalent bonding. The complexation depends on the size, shape and hydrophobicity of the guest molecule. $\beta$-Cyclodextrin catalyzes chemical reactions by supramolecular catalysis with high efficiency. No investi-gations of ring opening of epoxides with aromatic amines in the presence of $\beta$-cyclodextrin in water have been reported. Our earlier study on ester hydrolysis successfully catalyzed by $\beta$-cyclodextrin derivatives [21-23] promoted us to attempt ring opening of aromatic epoxides with aromatic amines in the presence of $\beta$-cyclodextrin in water.

\section{EXPERIMENTAL}

All the reactions were carried out without any special precautions in an atmosphere of air. Oxiranes and amines were purchased from Sigma-Aldrich. $\beta$-Cyclodextrin $(\beta-C D$, reagent grade) was recrystallized twice from $\mathrm{H}_{2} \mathrm{O}$ and dried in vacuo for $12 \mathrm{~h}$ at $100^{\circ} \mathrm{C}$. Water used as the solvent in all reactions was double distilled. ${ }^{1} \mathrm{H}$ NMR, ${ }^{13} \mathrm{C}$ NMR and HMBC spectra were recorded on BRUKER 500- or 400-MHz spectrometers. Highresolution mass spectra (HRMS) were observed on Bruker LC-Q-TOF spectrometer.

Synthesis of $\boldsymbol{\beta}$-amino alcohols: $\boldsymbol{\beta}$-Cyclodextrin $(0.50$ mmol) was dissolved in water $(15 \mathrm{~mL})$ by warming to $50^{\circ} \mathrm{C}$ until a clear solution was formed; then oxirane $(0.50 \mathrm{mmol})$ dissolved in acetone $(2 \mathrm{~mL})$ was added and the mixture was allowed to reach reaction temperature. Amine $(0.50 \mathrm{mmol})$ was added and the mixture was stirred at reaction temperature until the reaction was complete (TLC). The organic material was extracted with ethyl acetate. The organic layer was dried over anhydrous $\mathrm{Na}_{2} \mathrm{SO}_{4}$ and the solvent was removed under vacuum. The resulting crude product was further purified by silica-gel (60-120 mesh) column chromatography with petroleum ether:ethyl acetate (4:1) as eluent. The filtrate was evaporated to dryness under reduced pressure. The resulting residue was dissolved in a small amount of hot water and the aqueous solution was poured into ethyl acetate $(25 \mathrm{~mL})$ to give precipitate and 
filtrated to recover $\beta$-cyclodextrin (> $95 \%$ ), and recovered $\beta$-cyclodextrin was dried for $12 \mathrm{~h}$ at $95^{\circ} \mathrm{C}$ before reuse. NMR spectra of all compound $\beta$-amino alcohols were recorded in $\mathrm{CDCl}_{3}$ solvent. The new compounds 16B, 17B, 18B, 19B, 20B and 21B were characterized by ${ }^{1} \mathrm{H}$ NMR, ${ }^{13} \mathrm{C}$ NMR, HMBC and HRMS spectra.

\section{Spectral data}

1-Phenoxy-3-(phenylamino)propan-2-ol (1A) [Ref. 13]: ${ }^{1} \mathrm{H}$ NMR $\left(500 \mathrm{MHz}, \mathrm{CDCl}_{3}\right): \delta 7.29(\mathrm{dd}, J=8.5,7.5 \mathrm{~Hz}, 2 \mathrm{H})$, $7.18(\mathrm{dd}, J=8.4,7.5 \mathrm{~Hz}, 2 \mathrm{H}), 6.98(\mathrm{t}, J=7.4 \mathrm{~Hz}, 1 \mathrm{H}), 6.91$ (d, $J=7.9 \mathrm{~Hz}, 2 \mathrm{H}), 6.74(\mathrm{t}, J=7.3 \mathrm{~Hz}, 1 \mathrm{H}), 6.67(\mathrm{~d}, J=7.7 \mathrm{~Hz}$, $2 \mathrm{H}$ ), 4.24 (ddt, $J=8.4,7.1,4.2 \mathrm{~Hz}, 1 \mathrm{H}$ ), 4.04 (qd, $J=9.4,5.1$ $\mathrm{Hz}, 2 \mathrm{H}), 3.42(\mathrm{dd}, J=13.0,4.3 \mathrm{~Hz}, 1 \mathrm{H}), 3.28(\mathrm{dd}, J=13.0$, $7.2 \mathrm{~Hz}, 1 \mathrm{H}) .{ }^{13} \mathrm{C}$ NMR $\left(100 \mathrm{MHz}, \mathrm{CDCl}_{3}\right): \delta 158.69,148.34$, $129.85,129.60,121.61,118.33,114.84,113.57,70.32,69.10$, $46.91 \mathrm{ppm}$.

1-(o-Toluidino)-3-phenoxypropan-2-ol (2A) [Ref. 24]: ${ }^{1} \mathrm{H}$ NMR $\left(500 \mathrm{MHz}, \mathrm{CDCl}_{3}\right): \delta 7.32(\mathrm{dd}, J=21.1,12.8 \mathrm{~Hz}$, $2 \mathrm{H}), 7.16(\mathrm{t}, J=7.6 \mathrm{~Hz}, 1 \mathrm{H}), 7.11(\mathrm{~d}, J=7.3 \mathrm{~Hz}, 1 \mathrm{H}), 7.02(\mathrm{t}$, $J=7.4 \mathrm{~Hz}, 1 \mathrm{H}), 6.96(\mathrm{~d}, J=8.4 \mathrm{~Hz}, 2 \mathrm{H}), 6.72(\mathrm{dd}, J=17.4$, $7.7 \mathrm{~Hz}, 2 \mathrm{H}), 4.46-4.22(\mathrm{~m}, 1 \mathrm{H}), 4.13$ (qd, $J=9.4,5.0 \mathrm{~Hz}, 2 \mathrm{H})$, 3.52 (dd, $J=12.8,4.4 \mathrm{~Hz}, 1 \mathrm{H}), 3.38(\mathrm{dd}, J=12.8,7.0 \mathrm{~Hz}$, $1 \mathrm{H}), 2.61$ (brs, $1 \mathrm{H}), 2.21$ (s, 3H). ${ }^{13} \mathrm{C} \mathrm{NMR}\left(100 \mathrm{MHz}, \mathrm{CDCl}_{3}\right)$ : $\delta 158.41,146.00,130.26,129.61,127.17,122.71,121.37$, $117.65,114.55,110.11,70.21,68.76,46.60,17.52 \mathrm{ppm}$.

1-(p-Toluidino)-3-phenoxypropan-2-ol (3A) [Ref. 24]: ${ }^{1} \mathrm{H}$ NMR (500 MHz, $\left.\mathrm{CDCl}_{3}\right): \delta 7.38-7.30(\mathrm{~m}, 2 \mathrm{H}), 7.02(\mathrm{dd}$, $J=15.0,7.7 \mathrm{~Hz}, 3 \mathrm{H}), 6.98-6.92(\mathrm{~m}, 2 \mathrm{H}), 6.64(\mathrm{~d}, J=8.4 \mathrm{~Hz}$, $2 \mathrm{H}), 4.29$ (ddt, $J=8.3,7.2,4.2 \mathrm{~Hz}, 1 \mathrm{H}), 4.09$ (qd, $J=9.4,5.1$ $\mathrm{Hz}, 2 \mathrm{H}), 3.44(\mathrm{dd}, J=12.9,4.3 \mathrm{~Hz}, 1 \mathrm{H}), 3.31(\mathrm{dd}, J=12.9$, $7.2 \mathrm{~Hz}, 1 \mathrm{H}), 2.28(\mathrm{~s}, 3 \mathrm{H}) .{ }^{13} \mathrm{C} \mathrm{NMR}\left(100 \mathrm{MHz}, \mathrm{CDCl}_{3}\right): \delta 158.46$, $145.79,129.84,129.59,127.39,121.33,114.59,113.55,70.11$, 68.87, 47.10, $20.40 \mathrm{ppm}$.

1-(2-Methoxyphenylamino)-3-phenoxypropan-2-ol (4A) [Ref. 24]: ${ }^{1} \mathrm{H}$ NMR (500 MHz, $\mathrm{CDCl}_{3}$ ): $\delta 7.33$ (dd, $J=8.4,7.6$ $\mathrm{Hz}, 2 \mathrm{H}), 7.01(\mathrm{t}, J=7.4 \mathrm{~Hz}, 1 \mathrm{H}), 6.97(\mathrm{~d}, J=7.9 \mathrm{~Hz}, 2 \mathrm{H}), 6.91$ (td, $J=7.7,1.2 \mathrm{~Hz}, 1 \mathrm{H}), 6.85-6.78(\mathrm{~m}, 1 \mathrm{H}), 6.77-6.69(\mathrm{~m}, 2 \mathrm{H})$, 4.32 (ddd, $J=10.9,6.3,4.6 \mathrm{~Hz}, 1 \mathrm{H}), 4.11$ (qd, $J=9.4,5.1 \mathrm{~Hz}$, 2H), 3.88 (s, 3H), 3.49 (dd, $J=13.1,4.6 \mathrm{~Hz}, 1 \mathrm{H}), 3.37$ (dd, $J=$ 13.1, $6.9 \mathrm{~Hz}, 1 \mathrm{H}) .{ }^{13} \mathrm{C} \mathrm{NMR}\left(100 \mathrm{MHz}, \mathrm{CDCl}_{3}\right): \delta 158.50$, 147.18, 138.01, 129.57, 121.29, 121.28, 117.20, 114.60, 110.27, 109.63, 70.09, 68.89, 55.45, $46.56 \mathrm{ppm}$.

1-(4-Methoxyphenylamino)-3-phenoxypropan-2-ol (5A) [Ref. 25]: ${ }^{1} \mathrm{H}$ NMR (500 MHz, $\mathrm{CDCl}_{3}$ ): $\delta 7.33$ (dd, $J=8.5,7.5$ $\mathrm{Hz}, 2 \mathrm{H}), 7.01(\mathrm{t}, J=7.4 \mathrm{~Hz}, 1 \mathrm{H}), 6.95(\mathrm{~d}, J=7.9 \mathrm{~Hz}, 2 \mathrm{H})$, 6.86- $6.80(\mathrm{~m}, 2 \mathrm{H}), 6.71-6.65(\mathrm{~m}, 2 \mathrm{H}), 4.28$ (tt, $J=8.1,4.2$ $\mathrm{Hz}, 1 \mathrm{H}), 4.09(\mathrm{qd}, J=9.4,5.1 \mathrm{~Hz}, 2 \mathrm{H}), 3.78(\mathrm{~d}, J=4.0 \mathrm{~Hz}$, $3 \mathrm{H}), 3.41$ (dd, $J=12.8,4.2 \mathrm{~Hz}, 1 \mathrm{H}), 3.28(\mathrm{dd}, J=12.8,7.2$ $\mathrm{Hz}, 1 \mathrm{H}) .{ }^{13} \mathrm{C}$ NMR $\left(100 \mathrm{MHz}, \mathrm{CDCl}_{3}\right): \delta 158.45,152.68$, $142.18,129.59,121.33,114.97,114.84,114.58,70.14,68.86$, $55.82,47.81 \mathrm{ppm}$.

1-(2-Chlorophenylamino)-3-phenoxypropan-2-ol (6A) [Ref. 24]: ${ }^{1} \mathrm{H}$ NMR (500 MHz, $\mathrm{CDCl}_{3}$ ): $\delta$ 7.42-7.29 (m, 3H), $7.22-7.12(\mathrm{~m}, 1 \mathrm{H}), 7.02(\mathrm{t}, J=7.4 \mathrm{~Hz}, 1 \mathrm{H}), 6.96(\mathrm{~d}, J=7.9 \mathrm{~Hz}$, $2 \mathrm{H}), 6.81-6.74(\mathrm{~m}, 1 \mathrm{H}), 6.69(\mathrm{td}, J=7.7,1.3 \mathrm{~Hz}, 1 \mathrm{H}), 4.77$ (brs, $1 \mathrm{H}), 4.32(\mathrm{~d}, J=3.6 \mathrm{~Hz}, 1 \mathrm{H}), 4.12(\mathrm{qd}, J=9.4,5.0 \mathrm{~Hz}, 2 \mathrm{H})$, 3.47 (ddd, $J=19.9,13.0,5.5 \mathrm{~Hz}, 2 \mathrm{H}), 2.58(\mathrm{~d}, J=4.3 \mathrm{~Hz}$, 1H). $\left.{ }^{13} \mathrm{C} \mathrm{NMR} \mathrm{(100} \mathrm{MHz,} \mathrm{CDCl}_{3}\right): \delta 158.33,143.93,129.64$,
$129.32,127.87,121.43,119.73,117.85,114.55,111.49,69.93$, $68.68,46.35$ ppm.

1-(2-Nitrophenylamino)-3-phenoxypropan-2-ol (7A) [Ref. 24]: ${ }^{1} \mathrm{H}$ NMR (400 MHz, $\mathrm{CDCl}_{3}$ ): $\delta 8.34$ (brs, $\left.1 \mathrm{H}\right), 8.19$ $(\mathrm{dd}, J=8.6,1.6 \mathrm{~Hz}, 1 \mathrm{H}), 7.50-7.40(\mathrm{~m}, 1 \mathrm{H}), 7.37-7.27(\mathrm{~m}$, 2H), 7.03-6.90 (m, 4H), 6.68 (ddd, $J=8.4,7.0,1.1 \mathrm{~Hz}, 1 \mathrm{H}$ ), 4.32 (d, $J=16.6 \mathrm{~Hz}, 1 \mathrm{H}), 4.10$ (qd, $J=9.4,5.0 \mathrm{~Hz}, 2 \mathrm{H}), 3.71-$ $3.59(\mathrm{~m}, 1 \mathrm{H}), 3.59-3.48(\mathrm{~m}, 1 \mathrm{H}) .{ }^{13} \mathrm{C} \mathrm{NMR}\left(100 \mathrm{MHz}, \mathrm{CDCl}_{3}\right)$ : $\delta 158.16,145.52,136.26,129.66,127.02,121.59,115.79$, $114.55,113.75,69.68,68.61,45.58 \mathrm{ppm}$.

2-Phenyl-2-(phenylamino)ethanol (8B) [Ref. 26]: ${ }^{1} \mathrm{H}$ NMR (500 MHz, $\left.\mathrm{CDCl}_{3}\right): \delta 7.34(\mathrm{dt}, J=15.1,7.4 \mathrm{~Hz}, 4 \mathrm{H})$, 7.30-7.20 (m, 1H), 7.09 (t, $J=7.8 \mathrm{~Hz}, 2 \mathrm{H}), 6.67(\mathrm{t}, J=7.3 \mathrm{~Hz}$, $1 \mathrm{H}), 6.56(\mathrm{~d}, J=7.9 \mathrm{~Hz}, 2 \mathrm{H}), 4.48(\mathrm{dd}, J=6.8,4.3 \mathrm{~Hz}, 1 \mathrm{H})$, $3.91(\mathrm{dd}, J=11.1,4.2 \mathrm{~Hz}, 1 \mathrm{H}), 3.73(\mathrm{dd}, J=11.1,7.0 \mathrm{~Hz}, 1 \mathrm{H})$. ${ }^{13} \mathrm{C} \mathrm{NMR}\left(100 \mathrm{MHz}, \mathrm{CDCl}_{3}\right): \delta 147.86,140.45,129.14,128.26$, $126.79,117.92,113.90,67,38,59.87 \mathrm{ppm}$.

2-Phenyl-2-(o-tolylamino)ethan-1-ol (9B) [Ref. 27]: ${ }^{1} \mathrm{H}$ NMR (400 MHz, $\left.\mathrm{CDCl}_{3}\right): \delta$ 7.40-7.32 (m, 4H), 7.31-7.26 (m, $1 \mathrm{H}), 7.08$ (d, $J=7.3 \mathrm{~Hz}, 1 \mathrm{H}), 6.99-6.90(\mathrm{~m}, 1 \mathrm{H}), 6.65$ (td, $J=$ 7.4, $0.9 \mathrm{~Hz}, 1 \mathrm{H}), 6.39$ (d, $J=7.9 \mathrm{~Hz}, 1 \mathrm{H}), 4.56$ (dd, $J=7.0$, $4.2 \mathrm{~Hz}, 1 \mathrm{H}), 3.99$ (dd, $J=11.1,4.2 \mathrm{~Hz}, 1 \mathrm{H}), 3.80$ (dd, $J=11.1$, $7.0 \mathrm{~Hz}, 1 \mathrm{H}), 2.29$ (s, $3 \mathrm{H}) .{ }^{13} \mathrm{CNMR}\left(100 \mathrm{MHz}, \mathrm{CDCl}_{3}\right): \delta 145.13$, 140.20, 130.13, 128.88, 127.65, 127.01, 126.70, 122.61, 117.54, $111.51,67.55,59.80,17.72 \mathrm{ppm}$.

2-Phenyl-2-(p-tolylamino)ethan-1-ol (10B): ${ }^{1} \mathrm{H}$ NMR (400 MHz, CDCl $)$ : $\delta 7.49-7.18(\mathrm{~m}, 5 \mathrm{H}), 6.94(\mathrm{~d}, J=8.1 \mathrm{~Hz}, 2 \mathrm{H})$, $6.53(\mathrm{~d}, J=8.3 \mathrm{~Hz}, 2 \mathrm{H}), 4.51(\mathrm{dd}, J=7.1,4.2 \mathrm{~Hz}, 1 \mathrm{H}), 3.96$ (dd, $J=11.1,4.2 \mathrm{~Hz}, 1 \mathrm{H}), 3.77(\mathrm{dd}, J=11.1,7.2 \mathrm{~Hz}, 1 \mathrm{H}), 2.22$ (s, 3H). ${ }^{13} \mathrm{C}$ NMR $\left(100 \mathrm{MHz}, \mathrm{CDCl}_{3}\right): \delta 144.93,140.33,129.79$, $129.68,128.81,127.56,127.16,126.76,115.39,114.08,67.37$, 60.20, $20.38 \mathrm{ppm}$.

2-[(2-Methoxyphenyl)amino]-2-phenylethan-1-ol (11B) [Ref. 28]: ${ }^{1} \mathrm{H}$ NMR (400 MHz, $\mathrm{CDCl}_{3}$ ): $\delta$ 7.42-7.30 (m, 4H), 7.26 (ddd, $J=9.4,3.9,1.7 \mathrm{~Hz}, 1 \mathrm{H}), 6.79(\mathrm{dd}, J=7.7,1.5 \mathrm{~Hz}$, $1 \mathrm{H}), 6.75-6.60(\mathrm{~m}, 2 \mathrm{H}), 6.42(\mathrm{dd}, J=7.7,1.7 \mathrm{~Hz}, 1 \mathrm{H}), 4.54$ (dd, $J=7.3,4.3 \mathrm{~Hz}, 1 \mathrm{H}), 3.95$ (dd, $J=11.1,4.3 \mathrm{~Hz}, 1 \mathrm{H}$ ), 3.90 (s, 3H), 3.80 (dd, $J=11.1,7.3 \mathrm{~Hz}, 1 \mathrm{H}) .{ }^{13} \mathrm{C}$ NMR $(100 \mathrm{MHz}$, $\left.\mathrm{CDCl}_{3}\right): \delta 147.17,140.25,137.02,128.81,127.59,126.75$, $121.14,117.17,111.52,109.46,67.51,59.81,55.50 \mathrm{ppm}$.

2-[(4-Methoxyphenyl)amino]-2-phenylethan-1-ol (12B) [Ref. 25]: ${ }^{1} \mathrm{H} \mathrm{NMR}\left(500 \mathrm{MHz}, \mathrm{CDCl}_{3}\right): \delta$ 7.45-7.23 (m, 7H), $6.68(\mathrm{ddd}, J=8.4,7.0,1.1 \mathrm{~Hz}, 1 \mathrm{H}), 6.60(\mathrm{~d}, J=8.6 \mathrm{~Hz}, 1 \mathrm{H})$, $4.71(\mathrm{dd}, J=10.4,6.0 \mathrm{~Hz}, 1 \mathrm{H}), 4.18-4.00(\mathrm{~m}, 1 \mathrm{H}), 3.93(\mathrm{dd}, J=$ 11.1, $6.2 \mathrm{~Hz}, 1 \mathrm{H}), 2.20(\mathrm{~s}, 3 \mathrm{H}) .{ }^{13} \mathrm{C} \mathrm{NMR}\left(100 \mathrm{MHz}, \mathrm{CDCl}_{3}\right): \delta$ $152.43,141.32,140.32,128.81,128.57,128.04,127.59$, $126.75,126.07,115.31,114.78,67.37,60.87,55.71 \mathrm{ppm}$.

2-[(2-Chlorophenyl)amino]-2-phenylethan-1-ol (13B) [Ref. 29]: ${ }^{1} \mathrm{H}$ NMR (400 MHz, $\mathrm{CDCl}_{3}$ ): $\delta$ 7.56-7.20 (m, 6H), $6.96(\mathrm{td}, J=8.2,1.5 \mathrm{~Hz}, 1 \mathrm{H}), 6.61(\mathrm{td}, J=7.7,1.4 \mathrm{~Hz}, 1 \mathrm{H}), 6.44$ (dd, $J=8.2,1.3 \mathrm{~Hz}, 1 \mathrm{H}), 4.56(\mathrm{dd}, J=6.5,4.3 \mathrm{~Hz}, 1 \mathrm{H}), 3.99$ (dd, $J=11.1,4.1 \mathrm{~Hz}, 1 \mathrm{H}), 3.83(\mathrm{dd}, J=11.1,6.8 \mathrm{~Hz}, 1 \mathrm{H}) .{ }^{13} \mathrm{C} \mathrm{NMR}$ $\left(100 \mathrm{MHz}, \mathrm{CDCl}_{3}\right): \delta 143.06,139.52,129.14,128.95,127.82$, $127.68,126.68,119.76,117.86,112.86,67.38,59.67 \mathrm{ppm}$.

2-[(2-Nitrophenyl)amino]-2-phenylethan-1-ol (14B) [Ref. 24]: ${ }^{1} \mathrm{H} \mathrm{NMR}\left(400 \mathrm{MHz}, \mathrm{CDCl}_{3}\right): \delta 8.75(\mathrm{~d}, J=5.0 \mathrm{~Hz}$, $1 \mathrm{H}), 8.19(\mathrm{dd}, J=8.8,1.6 \mathrm{~Hz}, 1 \mathrm{H}), 7.41-7.28(\mathrm{~m}, 5 \mathrm{H}), 6.78-$ $6.52(\mathrm{~m}, 2 \mathrm{H}), 4.73(\mathrm{dt}, J=10.5,5.3 \mathrm{~Hz}, 1 \mathrm{H}), 4.04(\mathrm{dd}, J=11.1,4.0$ $\mathrm{Hz}, 1 \mathrm{H}), 3.94$ (dd, $J=11.1,6.3 \mathrm{~Hz}, 1 \mathrm{H}) .{ }^{13} \mathrm{C}$ NMR $(100 \mathrm{MHz}$, 
$\left.\mathrm{CDCl}_{3}\right): \delta 144.59,138.68,136.02,129.13,128.15,126.82,126.62$, $115.97,115.10,67.12,59.26 \mathrm{ppm}$.

2-(4-Chlorophenyl)-2-(phenylamino)ethanol (15B) [Ref. 19]: ${ }^{1} \mathrm{H} \mathrm{NMR}\left(500 \mathrm{MHz}, \mathrm{CDCl}_{3}\right): \delta 7.28(\mathrm{~d}, \mathrm{~J}=27.6 \mathrm{~Hz}$, $4 \mathrm{H}), 7.10(\mathrm{dd}, J=8.4,7.5 \mathrm{~Hz}, 2 \mathrm{H}), 6.69(\mathrm{t}, J=7.3 \mathrm{~Hz}, 1 \mathrm{H})$, $6.53(\mathrm{~d}, J=7.7 \mathrm{~Hz}, 2 \mathrm{H}), 4.46(\mathrm{dd}, J=7.0,4.1 \mathrm{~Hz}, 1 \mathrm{H}), 3.92(\mathrm{dd}$, $J=11.1,4.1 \mathrm{~Hz}, 1 \mathrm{H}), 3.71(\mathrm{dd}, J=11.1,7.0 \mathrm{~Hz}, 1 \mathrm{H}) .{ }^{13} \mathrm{C} \mathrm{NMR}$ $\left(100 \mathrm{MHz}, \mathrm{CDCl}_{3}\right): \delta 147.45,138.58,132.8,129.23,128.16$, 118.16, 113.88, 67.19, $59.34 \mathrm{ppm}$.

1-(Phenylamino)hexan-2-ol (22A) [Ref. 13]: ${ }^{1} \mathrm{H}$ NMR (400 MHz, $\left.\mathrm{CDCl}_{3}\right): \delta$ 7.27-7.14 (m, 2H), $6.73(\mathrm{t}, J=7.3 \mathrm{~Hz}$, $1 \mathrm{H}), 6.65(\mathrm{dd}, J=8.6,0.9 \mathrm{~Hz}, 2 \mathrm{H}), 3.97-3.59(\mathrm{~m}, 1 \mathrm{H}), 3.26$ $(\mathrm{dd}, J=12.9,3.2 \mathrm{~Hz}, 1 \mathrm{H}), 3.00(\mathrm{dd}, J=12.9,8.6 \mathrm{~Hz}, 1 \mathrm{H})$, $1.65-1.19(\mathrm{~m}, 6 \mathrm{H}), 0.93(\mathrm{t}, J=7.1 \mathrm{~Hz}, 3 \mathrm{H}) .{ }^{13} \mathrm{C}$ NMR $(100$ $\left.\mathrm{MHz}, \mathrm{CDCl}_{3}\right): \delta 148.33,129.33,117.91,113.31,70.41,50.34$, $34.83,27.83,22.78,14.08 \mathrm{ppm}$.

2-(4-Chlorophenyl)-2-(o-tolylamino)ethan-1-ol (16B): m.f. $\mathrm{C}_{15} \mathrm{H}_{16} \mathrm{NOCl}{ }^{1} \mathrm{H}$ NMR (400 MHz, $\mathrm{CDCl}_{3}$ ): $\delta$ 7.38-7.30 (m, 4H), $7.09(\mathrm{~d}, J=7.3 \mathrm{~Hz}, 1 \mathrm{H}), 7.01-6.94(\mathrm{~m}, 1 \mathrm{H}), 6.67(\mathrm{td}$, $J=7.4,0.9 \mathrm{~Hz}, 1 \mathrm{H}), 6.32(\mathrm{~d}, J=7.9 \mathrm{~Hz}, 1 \mathrm{H}), 4.50(\mathrm{dd}, J=7.2$, $4.1 \mathrm{~Hz}, 1 \mathrm{H}), 3.96(\mathrm{dd}, J=11.1,4.1 \mathrm{~Hz}, 1 \mathrm{H}), 3.75$ (dd, $J=11.1$, $7.2 \mathrm{~Hz}, 1 \mathrm{H}), 2.29$ (s, 3H). ${ }^{13} \mathrm{CNMR}\left(100 \mathrm{MHz}, \mathrm{CDCl}_{3}\right): \delta 144.89$, 138.86, 133.30, 130.23, 129.05, 128.12, 127.03, 122.74, 117.81, $111.52,67.34,59.33,17.70 \mathrm{ppm}$. HRMS (ESI) calculated for $\mathrm{C}_{15} \mathrm{H}_{16} \mathrm{NOCl}(\mathrm{M}+\mathrm{H})^{+} 262.0999$, found $(\mathrm{M}+\mathrm{H})^{+} 262.0995$.

2-(4-Chlorophenyl)-2-(p-tolylamino)ethan-1-ol (17B): m.f. $\mathrm{C}_{15} \mathrm{H}_{16} \mathrm{NOCl}$. ${ }^{1} \mathrm{H} \mathrm{NMR}\left(400 \mathrm{MHz}, \mathrm{CDCl}_{3}\right)$ : $\delta 7.30(\mathrm{~m}, 4 \mathrm{H})$, $6.93(\mathrm{~d}, J=8.0 \mathrm{~Hz}, 2 \mathrm{H}), 6.47(\mathrm{~d}, J=8.4 \mathrm{~Hz}, 2 \mathrm{H}), 4.44(\mathrm{dd}, J$ $=7.3,4.1 \mathrm{~Hz}, 1 \mathrm{H}), 3.90(\mathrm{dd}, J=11.1,4.1 \mathrm{~Hz}, 1 \mathrm{H}), 3.69(\mathrm{dd}, J$ $=11.1,7.3 \mathrm{~Hz}, 1 \mathrm{H}), 2.21(\mathrm{~s}, 3 \mathrm{H}) .{ }^{13} \mathrm{C}$ NMR $\left(100 \mathrm{MHz}, \mathrm{CDCl}_{3}\right)$ : $\delta 144.63,138.93,133.26,129.75,129.00,128.17,127.45$, $114.09,67.22,59.66,20.42 \mathrm{ppm}$. HRMS (ESI) calculated for $\mathrm{C}_{15} \mathrm{H}_{16} \mathrm{NOCl}(\mathrm{M}+\mathrm{H})^{+} 262.0999$, found $(\mathrm{M}+\mathrm{H})^{+} 262.0994$.

2-(4-Chlorophenyl)-2-[(2-methoxyphenyl)amino]ethan1-ol (18B): m.f. $\mathrm{C}_{15} \mathrm{H}_{16} \mathrm{NO}_{2} \mathrm{Cl}$. ${ }^{1} \mathrm{H}$ NMR (400 MHz, $\mathrm{CDCl}_{3}$ ): $\delta$ 7.30 (s, 4H), 6.79 (dd, $J=7.5,1.8 \mathrm{~Hz}, 1 \mathrm{H}), 6.75-6.63(\mathrm{~m}, 2 \mathrm{H})$, 6.34 (dd, $J=7.5,1.9 \mathrm{~Hz}, 1 \mathrm{H}), 4.49$ (dd, $J=7.3,4.3 \mathrm{~Hz}, 1 \mathrm{H}), 3.93$ (dd, $J=11.1,4.2 \mathrm{~Hz}, 1 \mathrm{H}), 3.90$ (s, $3 \mathrm{H}), 3.76(\mathrm{dd}, J=11.1,7.3 \mathrm{~Hz}$, 1H). $\left.{ }^{13} \mathrm{C} \mathrm{NMR} \mathrm{(100} \mathrm{MHz,} \mathrm{CDCl}_{3}\right): \delta 147.18,138.89,136.72$, 133.25, 128.98, 128.15, 121.12, 117.43, 111.50, 109.51, 67.31, 59.31, 55.50 ppm. HRMS (ESI) calculated for $\mathrm{C}_{15} \mathrm{H}_{16} \mathrm{NO}_{2} \mathrm{Cl}$ $(\mathrm{M}+\mathrm{H})^{+}$278.0948, found $(\mathrm{M}+\mathrm{H})^{+} 278.0945$.
2-(4-Chlorophenyl)-2-[(4-methoxyphenyl)amino]ethan1-ol (19B): m.f. $\mathrm{C}_{15} \mathrm{H}_{16} \mathrm{NO}_{2} \mathrm{Cl}$. ${ }^{1} \mathrm{H} \mathrm{NMR}\left(400 \mathrm{MHz}, \mathrm{CDCl}_{3}\right)$ : $\delta$ 7.30 (s, 4H), 6.79-6.61 (m, 2H), 6.55-6.43 (m, 2H), 4.39 (dd, $J=7.5,4.1 \mathrm{~Hz}, 1 \mathrm{H}), 3.89(\mathrm{dd}, J=11.1,4.1 \mathrm{~Hz}, 2 \mathrm{H}), 3.70(\mathrm{~s}, 3 \mathrm{H})$, 3.69-3.64 (m, 1H). ${ }^{13} \mathrm{C}$ NMR (100 MHz, $\left.\mathrm{CDCl}_{3}\right): \delta 152.53$, 141.03, 138.97, 133.28, 129.00, 128.19, 115.32, 114.80, 67.22, $60.31,55.73$ ppm. HRMS (ESI) calculated for $\mathrm{C}_{15} \mathrm{H}_{16} \mathrm{NO}_{2} \mathrm{Cl}$ $(\mathrm{M}+\mathrm{H})^{+} 278.0948$, found $(\mathrm{M}+\mathrm{H})^{+} 278.0944$.

2-(4-Chlorophenyl)-2-[(2-chlorophenyl)amino]ethan1-ol (20B): m.f. $\mathrm{C}_{14} \mathrm{H}_{13} \mathrm{NOCl}_{2}{ }^{1} \mathrm{H} \mathrm{NMR}\left(500 \mathrm{MHz}, \mathrm{CDCl}_{3}\right)$ : $\delta$ $7.32(\mathrm{dt}, J=11.3,8.6 \mathrm{~Hz}, 5 \mathrm{H}), 6.99(\mathrm{t}, J=7.6 \mathrm{~Hz}, 1 \mathrm{H}), 6.65(\mathrm{t}$, $J=7.5 \mathrm{~Hz}, 1 \mathrm{H}), 6.40(\mathrm{~d}, J=8.0 \mathrm{~Hz}, 1 \mathrm{H}), 5.19(\mathrm{~s}, 1 \mathrm{H}), 4.54(\mathrm{~s}, 1 \mathrm{H})$, 4.20-3.92 (m, 1H), $3.82(\mathrm{dd}, J=10.8,7.0 \mathrm{~Hz}, 1 \mathrm{H}) .{ }^{13} \mathrm{C} \mathrm{NMR}$ $\left(100 \mathrm{MHz}, \mathrm{CDCl}_{3}\right): \delta 142.79,138.17,133.53,129.20,129.10$, 128.06, 127.66, 119.84, 118.11, 112.80, 67.14, 59.15 ppm. HRMS (ESI) calculated for $\mathrm{C}_{14} \mathrm{H}_{13} \mathrm{NOCl}_{2}(\mathrm{M}+\mathrm{H})^{+} 282.0452$, found $(\mathrm{M}+\mathrm{H})^{+} 282.0449$.

2-(4-Chlorophenyl)-2-[(2-nitrophenyl)amino]ethan-1ol (21B): m.f. $\mathrm{C}_{14} \mathrm{H}_{13} \mathrm{~N}_{2} \mathrm{O}_{3} \mathrm{Cl}$. ${ }^{1} \mathrm{H}$ NMR (400 MHz, $\mathrm{CDCl}_{3}$ ): $\delta$ $8.73(\mathrm{~d}, J=5.4 \mathrm{~Hz}, 1 \mathrm{H}), 8.21$ (dd, $J=8.6,1.5 \mathrm{~Hz}, 1 \mathrm{H}), 7.47-7.26$ (m, 4H), 6.69 (ddd, $J=8.4,7.0,1.1 \mathrm{~Hz}, 1 \mathrm{H}), 6.60(\mathrm{~d}, J=8.6 \mathrm{~Hz}$, $1 \mathrm{H}), 4.71(\mathrm{dd}, J=10.5,6.0 \mathrm{~Hz}, 1 \mathrm{H}), 4.18-4.00(\mathrm{~m}, 1 \mathrm{H}), 3.93$ $(\mathrm{dd}, J=11.1,6.2 \mathrm{~Hz}, 1 \mathrm{H}) .{ }^{13} \mathrm{CNMR}\left(400 \mathrm{MHz}, \mathrm{CDCl}_{3}\right): \delta 144.29$, 137.36, 136.07, 129.31, 128.02, 126.89, 116.23, 114.96, 99.99, 66.90, 58.68 ppm. HRMS (ESI) calculated for $\mathrm{C}_{14} \mathrm{H}_{13} \mathrm{~N}_{2} \mathrm{O}_{3} \mathrm{Cl}$ $[\mathrm{M}+\mathrm{Na}]^{+}$315.0512, found $[\mathrm{M}+\mathrm{Na}]^{+}$315.0510.

\section{RESULTS AND DISCUSSION}

Initially, for the model study, the ring opening reaction of 1,2-epoxy-3-phenoxy propane was carried out with aniline in water using $\beta$-cyclodextrin as the catalyst (Table-1). The yield was only $78 \%$ after stirring for $8 \mathrm{~h}$ under $20{ }^{\circ} \mathrm{C}$ (Table-1, entry 1). While the yield was up to $96 \%$ when the temperature was $30^{\circ} \mathrm{C}$ and the yields were more than $95 \%$ when the temperature was higher than $30^{\circ} \mathrm{C}$ (Table-1, entries 2-6). However, the ratio of the major isomer A decreased as the temperature increased. The higher regioselectivity was achieved at 20 and $30{ }^{\circ} \mathrm{C}$ (Table-1, entries 2, 3). In order to understand the role of catalyst, control reaction was proceeded at $30{ }^{\circ} \mathrm{C}$ (Table-1, entry 7) and only $9 \%$ of the product was obtained even with prolonged reaction time in the absence of catalyst.

TABLE-1

RING-OPENING REACTION OF 1,2-EPOXY-3-PHENOXY PROPANE WITH ANILINE IN THE PRESENCE OF CYCLODEXTRIN AT DIFFERENT REACTION CONDITIONS
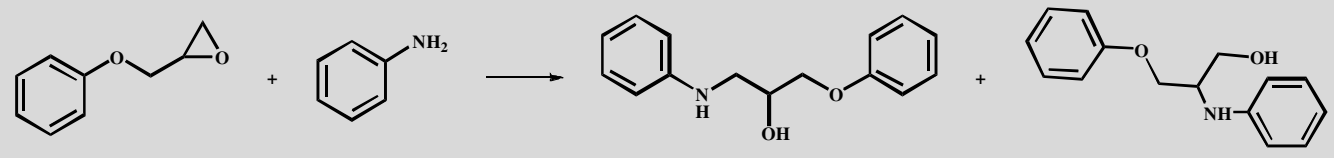

A

B

\begin{tabular}{ccccc}
\hline Entry & Temperature $\left({ }^{\circ} \mathrm{C}\right)$ & Time $(\mathrm{h})$ & ${\text { Yield }(\%)^{\mathrm{b}}}$ & Ratio $(\mathrm{A}: \mathrm{B})^{\mathrm{c}}$ \\
\hline 1 & 20 & 8 & 78 & $85: 15$ \\
2 & 30 & 8 & 96 & $85: 15$ \\
3 & 40 & 8 & 96 & $82: 18$ \\
4 & 50 & 8 & 95 & $73: 27$ \\
5 & 60 & 6 & 96 & $72: 28$ \\
6 & 80 & 5 & 9 & $65: 35$ \\
7 & $30^{\mathrm{a}}$ & 16 & $84: 16$ \\
\hline
\end{tabular}

${ }^{\mathrm{a}}$ No cyclodextrin; ${ }^{\mathrm{b}}$ Isolated yield; ${ }^{\mathrm{c}}$ The ratio of isomers was calculated by ${ }^{1} \mathrm{H}$ NMR 
With the optimized reaction conditions, the scope of the reactions was investigated at $30^{\circ} \mathrm{C}$ with the styrene oxide, 4chlorostyrene oxide, 1,2-epoxy-3-phenoxy propane and 1,2epoxyhexane as the substrates and series of aromatic amines with electron-donating and electron-withdrawing groups as the nucleophiles. As listed in Table-2, $\beta$-amino alcohols were obtained in high yield (80-96\%). For amines with an electronwithdrawing group, moderate yields were obtained (Table-2, entries $6,13,20)$. However, for 2-nitroaniline, the yields were low even after prolong the reaction time (Table-2, entries 7 , 14, 21). Similar to 1,2-epoxy-3-phenoxy propane, the yields of ring-opening of Styrene oxide, 4-chlorostyrene oxide and 1,2 -epoxyhexane with aniline were also very low $(<10 \%)$ in the absence of cyclodextrin.

Unsymmetrical alkyl oxiranes such as 1,2-epoxy-3-phenoxy propane and 1,2-epoxyhexane afforded $\beta$-amino alcohols with preferential attack at the terminal position. For anilines with ortho-substituents, only one isomer was obtained (Table-2,

TABLE-2

RING-OPENING REACTION OF EPOXIDES WITH AROMATIC AMINES IN THE PRESENCE OF $\beta$-CYCLODEXTRIN

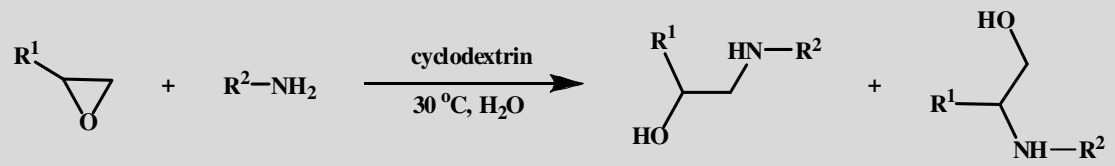

A

B

\begin{tabular}{|c|c|c|c|c|c|}
\hline Entry & Epoxide & Amine & Time (h) & Yield $(\%)^{\mathrm{a}, \mathrm{b}}$ & Ratio $(\mathrm{A}: \mathrm{B})^{\mathrm{c}}$ \\
\hline 1 & & & 8 & 96 & $85: 15$ \\
\hline 2 & & & 7 & 84 & 100:0 \\
\hline 3 & & & 7 & 87 & 83:17 \\
\hline 4 & & & 6 & 86 & 100:0 \\
\hline 5 & & & 6 & 90 & $82: 18$ \\
\hline 6 & & & 24 & 70 & 100:0 \\
\hline 7 & & & 24 & 20 & 100:0 \\
\hline 8 & & & 7 & 90 & 7:93 \\
\hline 9 & & & 7 & 83 & 3:97 \\
\hline 10 & & & 11 & 88 & $0: 100$ \\
\hline 11 & & & 8 & 93 & 10:90 \\
\hline
\end{tabular}




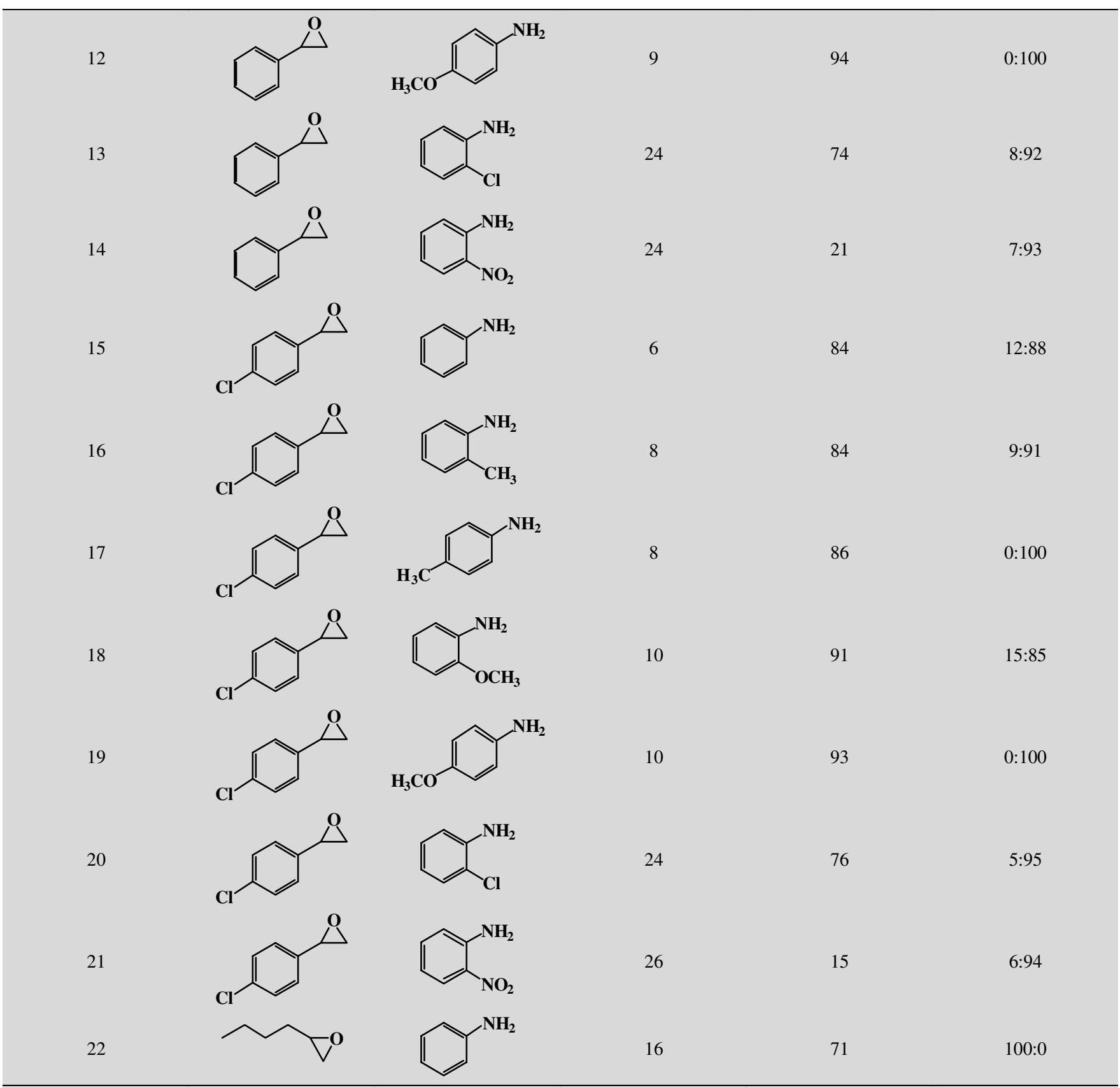

${ }^{a}$ All products were characterized by ${ }^{1} \mathrm{H}$ NMR and ${ }^{13} \mathrm{C}$ NMR; ${ }^{b}$ Isolated yield; ${ }^{c}$ The ratio of isomers were calculated by ${ }^{1} \mathrm{HNMR}$

entries $2,4,6,7,22)$. In the case of aniline, 4-methylaniline and 4-methoxyaniline, a mixture of two regioisomers was isolated in ratios of $85: 15,83: 17$ and $82: 18$, respectively. (Table-2, entries 1,3,5).Alkenyl oxiranes such as styrene oxide and ( $p$-chlorophenyl) styrene oxide have been treated with various amines in the presence of $\beta$-cyclodextrin. For most of the amines, only one isomer was obtained (Table-2, entries $10,12,13,14,17,19)$ and for anilines, anilines with orthosubstituents, a mixture of two regioisomers was produced (Table-2, entries 8, 9, 11, 15, 16, 18, 20, 21).

According to the literature [30], benzyl ring of the epoxides insert into the cavity of $\beta$-cyclodextrin partially from the secondary rim and the epoxides are fixed through supramolecular interaction. Moreover, hydrogen bond is formed between the hydroxyl group of $\beta$-cyclodextrin and the oxygen atom of epoxides. Hydrogen bond favours nucleophiles to attack the
C-O bond of epoxide. For alkyl oxirane 1,2-epoxy-3-phenoxy propane, terminal carbon atom is attacked more preferentially. Steric effect may play important roles on the ring-opening reaction. As the anilines with bukly ortho-substituents, only one isomer was obtained. In the case of smaller steric-hindrance of aniline and para-substituent anilines, mixed products were obtained. For styrene oxide and ( $p$-chlorophenyl) styrene oxide, the $\mathrm{C}-\mathrm{O}$ is activated by the hydrogen bond. Benzylic carbon atom is attacked easily due to its high activity. So electronic effect may affect this transformation deeply. Aromatic amines with the substituent on the para-position attack the benzylic carbon completely. The reaction intermediates of the ring-opening catalyzed by $\beta$-cyclodextrin were proposed in Scheme-I.

In order to evaluate the recycling capability of $\beta$-cyclodextrin, the ring opening of 1,2-epoxy-3-phenoxy propane with aniline was carried out with recovered catalyst. As revealed in 


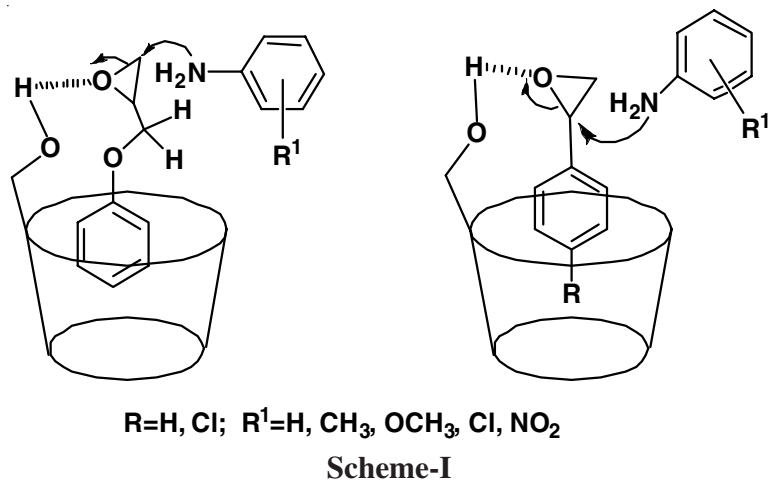

Table-3, the recovered catalyst showed almost equal efficiency in consecutive four cycles under identical reaction conditions and all the ratios of two regioisomers A:B are 85:15, which indicated that the catalyst was stable and suitable to be reused for several times.

\begin{tabular}{|c|c|c|c|c|}
\hline \multicolumn{5}{|c|}{$\begin{array}{c}\text { TABLE-3 } \\
\text { REUSE OF CYCLODEXTRIN FOR THE RING- } \\
\text { OPENING REACTION OF 1,2-EPOXY-3-PHENOXY } \\
\text { PROPANE WITH ANILINE }\end{array}$} \\
\hline Cycle & 1 & 2 & 3 & 4 \\
\hline Yield $(\%)^{\mathrm{a}}$ & 96 & 95 & 95 & 94 \\
\hline
\end{tabular}

\section{Conclusion}

In conclusion, $\beta$-cyclodextrin is a highly efficient and reusable catalyst for the ring-opening of epoxides containing benzene group with different aniline derivatives. Excellent yields were achieved for this reaction along with high regioselectivity. This method is simple, economic, environmental and efficient, which would be a useful way to modern synthetic methodology in green chemistry.

\section{ACKNOWLEDGEMENTS}

This work was supported by the National Natural Science Foundation of China (Nos. 21102040, 21472038,21202041 and 21505035), the Key Project of science and technology program of Hunan Provincial Education Department of China (No.15A027), Key Laboratory of Functional Organometallic Materials of Hunan Province.

\section{REFERENCES}

1. P. O'Brien, Angew. Chem. Int. Ed., 38, 326 (1999); https://doi.org/10.1002/(SICI)1521-3773(19990201)38:3<326::AIDANIE326>3.0.CO;2-T.

2. G. Li, H.T. Chang and K.B. Sharpless, Angew. Chem. Int. Ed. Engl., 35, 451 (1996); https://doi.org/10.1002/anie.199604511.

3. D.J. Ager, I. Prakash and D.R. Schaad, Chem. Rev., 96, 835 (1996); https://doi.org/10.1021/cr9500038.

4. J.A. Deyrup and C.L. Moyer, J. Org. Chem., 34, 175 (1969); https://doi.org/10.1021/jo00838a038.

5. C.L. Kissel and B. Rickborn, J. Org. Chem., 37, 2060 (1972); https://doi.org/10.1021/j000978a002.

6. M.C. Carre, J.P. Houmounou and P. Caubere, Tetrahedron Lett., 26, 3107 (1985);

https://doi.org/10.1016/S0040-4039(00)98630-1.
7. J. Yamada, M. Yumoto and Y. Yamamoto, Tetrahedron Lett., 30, 4255 (1989);

https://doi.org/10.1016/S0040-4039(01)80704-8.

8. J. Auge and F. Leroy, Tetrahedron Lett., 37, 7715 (1996); https://doi.org/10.1016/0040-4039(96)01731-5.

9. M. Chini, P. Crotti, L. Favero, F. Macchia and M. Pineschi, Tetrahedron Lett., 35, 433 (1994); https://doi.org/10.1016/0040-4039(94)85073-9.

10. A.T. Placzek, J.L. Donelson, R. Trivedi, R.A. Gibbs and S.K. De, Tetrahedron Lett., 46, 9029 (2005); https://doi.org/10.1016/j.tetlet.2005.10.106.

11. G. Sundararajan, K. Vijayakrishna and B. Varghese, Tetrahedron Lett., 45, 8253 (2004); https://doi.org/10.1016/j.tetlet.2004.09.023.

12. A.K. Chakraborti and A. Kondaskar, Tetrahedron Lett., 44, 8315 (2003); https://doi.org/10.1016/j.tetlet.2003.09.046.

13. N.R. Swamy, T.V. Goud, S.M. Reddy, Y. Venkateswarlu and P. Krishnaiah, Synth. Commun., 34, 727 (2004);

https://doi.org/10.1081/SCC-120027721.

14. L.D. Pachón, P. Gamez, J.J.M. van Brussel and J. Reedijk, Tetrahedron Lett., 44, 6025 (2003); https://doi.org/10.1016/S0040-4039(03)01480-1.

15. N. Tan, S. Yin, Y. Li, R. Qiu, Z. Meng, X. Song, S. Luo, C.-T. Au and W.-Y. Wong, J. Organomet. Chem., 696, 1579 (2011); https://doi.org/10.1016/j.jorganchem.2010.12.035.

16. A. Kamal, R. Ramu, M.A. Azhar and G.B.R. Khanna, Tetrahedron Lett., 46, 2675 (2005); https://doi.org/10.1016/j.tetlet.2005.02.073.

17. J.S. Yadav, B.V.S. Reddy, A.K. Basak and A.V. Narsaiah, Tetrahedron Lett., 44, 1047 (2003); https://doi.org/10.1016/S0040-4039(02)02735-1.

18. S.R. Kumar and P. Leelavathi, J. Mol. Catal. Chem., 266, 65 (2007); https://doi.org/10.1016/j.molcata.2006.10.045.

19. S.S. Chimni, N. Bala, V.A. Dixit and P.V. Bharatam, Tetrahedron, 66 , $3042(2010)$ https://doi.org/10.1016/j.tet.2010.02.053.

20. R.I. Kureshy, S. Agrawal, M. Kumar, N.H. Khan, S.H.R. Abdi and H.C. Bajaj, Catal. Lett., 134, 318 (2010); https://doi.org/10.1007/s10562-009-0237-z.

21. S.P. Tang, P. Hu, H.Y. Chen, S. Chen, Z.W. Mao and L.N. Ji, J. Mol. Catal. Chem., 335, 222 (2011); https://doi.org/10.1016/j.molcata.2010.11.037.

22. S.-P. Tang, S. Chen, G.-F. Wu, H.-Y. Chen, Z.-W. Mao and L.-N. Ji, Inorg. Chem. Commun., 14, 184 (2011); https://doi.org/10.1016/j.inoche.2010.10.018

23. S.-P. Tang, Y.-H. Zhou, H.-Y. Chen, C.-Y. Zhao, Z.-W. Mao and L.-N. Ji, Chem. Asian J., 4, 1354 (2009); https://doi.org/10.1002/asia.200900108.

24. M. Moghadam, S. Tangestaninejad, V. Mirkhani, I. Mohammadpoor-Baltork, S. Gorjipoor and P. Yazdani, Synth. Commun., 39, 552 (2009); https://doi.org/10.1080/00397910802406729.

25. M. Maheswara, K.S.V.K. Rao and J.Y. Do, Tetrahedron Lett., 49, 1795 (2008); https://doi.org/10.1016/j.tetlet.2008.01.044.

26. M. Vijender, P. Kishore, P. Narender and B. Satyanarayana, J. Mol. Catal. Chem., 266, 290 (2007); https://doi.org/10.1016/j.molcata.2006.09.006.

27. G.R. Krishnan, K.S. Kajal and K. Sreekumar, Monatsh. Chem., 143, 637 (2012); https://doi.org/10.1007/s00706-011-0628-x.

28. L.R. Reddy, M.A. Reddy, N. Bhanumathi and K.R. Rao, New J. Chem., 25, 221 (2001); https://doi.org/10.1039/b0077291.

29. A. Kamal, B.R. Prasad, A.M. Reddy and M.N.A. Khan, Catal. Commun., 8, 1876 (2007); https://doi.org/10.1016/j.catcom.2007.02.029.

30. R. Sridhar, B. Srinivas, K. Surendra, N.S. Krishnaveni and K.R. Rao, Tetrahedron Lett., 46, 8837 (2005); https://doi.org/10.1016/j.tetlet.2005.10.094. 